\title{
Purification of single photons by temporal heralding of quantum dot sources
}

\author{
Hamza Abudayyeh, ${ }^{\dagger}$ Boaz Lubotzky, ${ }^{\dagger}$ Somak Majumder ${ }^{\ddagger}$ Jennifer A. \\ Hollingsworth, ${ }^{\ddagger}$ and Ronen Rapaport ${ }^{*}$, \\ $\dagger$ Racah Institute for Physics, the Applied Physics Department and the Center for \\ Nanoscience and Nanotechnology, The Hebrew University of Jerusalem, Jerusalem \\ 9190401, Israel \\ $\ddagger$ Materials Physics \& Applications Division: Center for Integrated Nanotechnologies, Los \\ Alamos National Laboratory, Los Alamos, New Mexico 87545, United States \\ \Racah Institute for Physics and Center for Nanoscience and Nanotechnology, The Hebrew \\ University of Jerusalem, Jerusalem 9190401, Israel \\ E-mail: ronenr@phys.huji.ac.il
}

\begin{abstract}
Efficient, high rate photon sources with high single photon purity are essential ingredients for quantum technologies. Single photon sources based on solid state emitters such as quantum dots are very advantageous for integrated photonic circuits, but they can suffer from a high two-photon emission probability, which in cases of non-cryogenic environment cannot be spectrally filtered. Here we propose two temporal purificationby-heralding methods for using a two photon emission process to yield highly pure and efficient single photon emission, bypassing the inherent problem of spectrally overlapping bi-photon emission at elevated temperatures. We experimentally emulate their feasibility on the emission from a single nanocrystal quantum dot at room temperature,
\end{abstract}


exhibiting single photon purities exceeding 99.5\%, without a significant loss of single photon efficiency. By utilizing these methods with commercially available components, nanocrystal quantum dots can be made realistic high-quality room-temperature sources for single photons. These approaches can also be applied for any indeterministic source of spectrally broadband photon pairs.

\section{Introduction:}

A single photon source (SPS) is proposed as a crucial resource for several major applications in quantum technologies. ${ }^{1-3}$ The required performance of such a source depends on the application it would be used for, ${ }^{3}$ however generally most applications require stable, compact, high-rate and high-single-photon-purity SPSs. The most widely used resource for single photons comes from low efficiency down-converted photon-pairs from non-linear processes in solids. ${ }^{4-7}$ Recently however, research has been focused on developing more efficient solid state SPSs such as semiconductor self assembled quantum dots (QDs), ${ }^{8-12}$ chemically synthesized semiconductor nanocrystal quantum dots (NQDs), ${ }^{13-18}$ color centers in crystals, ${ }^{19-21}$ carbon nanotubes, ${ }^{22,23}$ and defect states in two-dimensional materials ${ }^{24,25}$ among others.

One crucial requirement from an SPS is a very high single photon purity, i.e., a high probability that an emission event contains a single photon. Unfortunately most solid-state SPSs, especially room-temperature sources, do not inherently have sufficiently high purities. QD-based SPSs which operate at cryogenic temperatures, for example, have resolvable multi-exciton spectral lines. Therefore multi-exciton emission can be spectrally filtered leaving only single photon emission from the single exciton state (X). However, the necessity of a cryogenic work environment is limiting technologies, and semiconductor QD sources working at elevated temperatures have only been achieved with either wide bandgap QDs, ${ }^{26}$ or using the smaller NQDs. ${ }^{13,14}$ Yet, operating at elevated temperatures introduces a significant limitation in maintaining both high purity together with a high source efficiency due to the spectral overlap between the single exciton (X) and bi-exciton (BX) emission. The severity 
of BX emission increases significantly when the Auger recombination processes ${ }^{27}$ are suppressed to increase QD stability or when NQDs are radiatively enhanced to achieve higher rates. ${ }^{28-30}$ This detrimental problem of reduced purity is rather inherent for such elevated temperature SPSs, and no solution to increase purity without a significant sacrifice in terms of brightness has been successfully proposed or implemented to the best of our knowledge.

Here we propose and demonstrate two temporal purification-by-heralding methods for using a two photon emission process to yield highly pure and efficient single photon emission, thus bypassing the inherent problem of spectrally overlapping bi-photon emission. We then experimentally emulate these temporal purification methods on the emission from a single NQD that is particularly prone to efficient BX emission, ${ }^{31}$ and show that employing such techniques enable reaching single photon purities exceeding $99.5 \%$, without a significant loss of single photon efficiency.

\section{Purification by Temporal Heralding:}

Chemically synthesized NQDs (see Fig. 1a) are an excellent exemplary system to demonstrate and apply the temporal photon purification and heralding concepts. NQDs are very promising for integrated and efficient SPSs at room temperature. ${ }^{32-36}$ Unfortunately, it turns out that the photostability (in terms of photo-blinking) of an NQD can be intimately related to its BX quantum yield $\left(\mathrm{QY}_{B X}\right)$ defined as the probability that the BX would radiatively decay to the $\mathrm{X}$ state. A key mechanism responsible for photo-blinking involves Augermediated non-radiative recombination of charged excitons. ${ }^{27}$ Thus, as the stability of the source against photo-blinking increases, $\mathrm{QY}_{B X}$ also usually increases due to the suppression of Auger recombination processes, ${ }^{27}$ leading to a dramatic drop of both the source efficiency $\left(\eta=P_{1}\right)$ and purity $\left(S=P_{1} /\left(P_{1}+P_{2}\right)\right)$, where $P_{1}$ and $P_{2}$ are the probability of emitting one and two photons respectively (see Fig. 1b). Higher order multi-exciton emission is usually negligible in these quantum dots due their strong excitation power dependence and due to 
the cubic (or nearly cubic) scaling of the Auger recombination rate with exciton multiplicity whereas the radiative decay rate only has a quadratic scaling. ${ }^{37}$ Furthermore the emission of these multi-excitons can be spectrally separated from the exciton line at room temperature. ${ }^{37}$ Thus NQD typically emits at most two photons per excitation cycle from the BX and $\mathrm{X}$ state with probabilities given by the $\mathrm{BX}$ and $\mathrm{X}$ quantum yields $\left(\mathrm{QY} \mathrm{BX}_{X}\right.$ and $\mathrm{QY}_{X}$ respectively). Notably a similar increase in $\mathrm{QY}_{B X}$ has been reported when NQDs were coupled to plasmonic emission rate enhancers,${ }^{28-30}$ showing that trying to increase the inherent emission rate of an NQD via the Purcell effect has a detrimental effect on the purity of the source. Therefore it seems necessary to develop some photon purification methods that will enable utilizing NQDs as efficient high-quality room temperature SPSs.
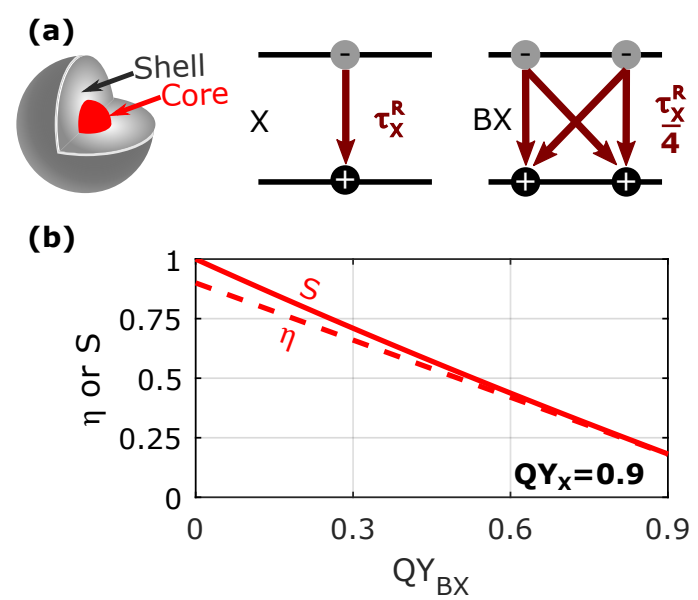

Figure 1: (a) Illustration of a spherical nanocrystal quantum dot displaying the radiative recombination pathways available for the $\mathrm{X}$ and BX state. (b) Single photon purity (solid line) and efficiency (dashed line) for a quantum dot as a function of the BX quantum yield.

Since neither spectral nor polarization based filtering of the BX emission is possible, a different approach is essential. One such approach, referred to here as time-gated filtering (TGF), is available in the time domain by using the difference in the lifetimes between the $\mathrm{X}$ and BX to temporally filter out (and discard!) the BX emission in a time-resolved measurement (see Fig. 1a). ${ }^{38,39}$ In this approach however, due to the overlap between the BX and X decays, a very unfavorable trade-off between the achievable purity and efficiency appears for high BX and X QYs. ${ }^{40}$ For example, in the theoretical limit when $Q Y_{X}=$ 

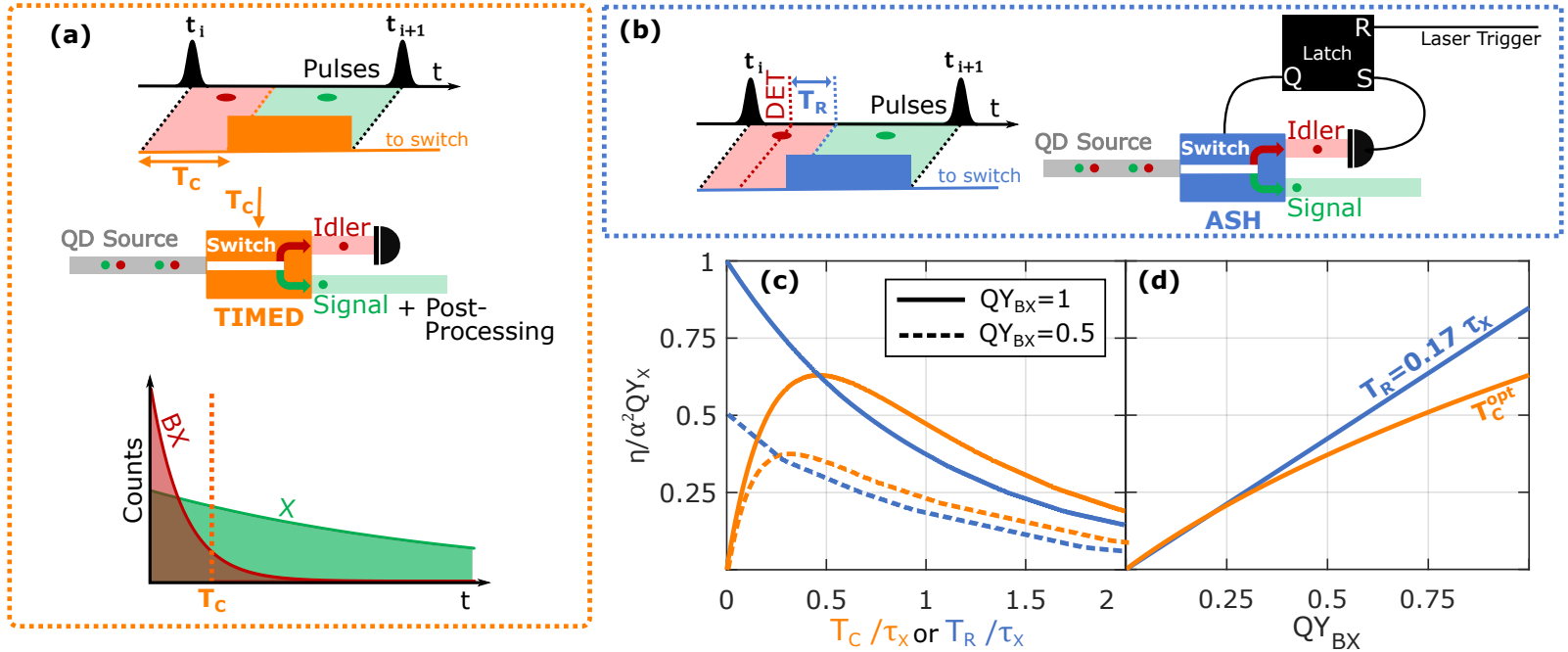

Figure 2: Schematic representations of the TIMe-resolved heraldED (TIMED), and (b) Active Switching Heralded (ASH) techniques proposed in this work. (c) Theoretical calculations of the heralding efficiency as a function of the cutoff time $T_{C}$ (for TIMED) or the response time $T_{R}$ (for ASH) normalized by the collection efficiency and the exciton quantum yield. Solid lines correspond to unity BX quantum yield whereas dashed lines correspond to $Q Y_{B X}=0.5$. (d) Theoretical normalized heralding efficiency using optimal parameters as a function of the BX quantum yield assuming unity X quantum yield.

$Q Y_{B X}=1$ obtaining a purity of $99.5 \%$ would entail operating at an efficiency of about $25 \%$ even if the collection efficiency of the system, $\alpha$, is unity. ${ }^{40}$ For currently available highquality high-rate NQD emitters having $Q Y_{X}=0.61, Q Y_{B X}=0.7,{ }^{18}$ the efficiency would only be $8 \%$ for $\alpha=1$. Therefore such an approach is not so useful for a practical high-quality SPS which requires both $\eta$ and $S$ to approach unity.

An alternative approach would be to use the two photon events in a heralded scheme to produce highly pure single photon states. The simplest possible heralding approach, referred to here as the beam splitter heralded scheme, would be to use a 50:50 beam splitter that would randomly direct the emission either to an idler port or a signal port. In a post processing step only signal photons heralded by an idler photon would be chosen, assuring $S=1$ in the signal channel. This technique however has a maximum theoretical efficiency of $\eta=0.5$ in the ideal case where $Q Y_{X}=Q Y_{B X}=1$ and $\alpha=1$, due to the random routing of the beam splitter. 
In order to overcome all these limitations we propose two main purification heralded schemes that are illustrated in Fig. 2a and b.

\section{Passive Heralding:}

The first scheme, called the TIMe-resolved heraldED (TIMED) technique, utilizes the large difference between the BX and X spontaneous radiative lifetimes, and their cascaded emission nature to implement a time gate on an optical switch (see Fig. 2a). In this scheme, an optical switch with a fixed time gate will replace the 50:50 beam splitter. The switch will be open to the idler port from the beginning of the excitation cycle up to a fixed cutoff time $\left(T_{C}\right)$. For $t>T_{C}$ the switch will direct the photons to the signal port until the the next excitation cycle. ${ }^{41}$ Here again, only signal photons that were preceded by an idler photon detection would be chosen in a post-processing step. The single photon efficiency in this scheme is given by: ${ }^{40}$

$$
\begin{aligned}
\eta_{T I M E D}= & \alpha^{2} Q Y_{X} Q Y_{B X} \frac{\tau_{X}}{\tau_{X}-\tau_{B X}} \times \\
& \times\left[\exp \left(-T_{C} / \tau_{X}\right)-\exp \left(-T_{C} / \tau_{B X}\right)\right],
\end{aligned}
$$

where $\tau_{X}$ and $\tau_{B X}$ are the $\mathrm{X}$ and BX lifetimes respectively. As seen in Fig. 2c there is an optimal gate time $T_{C}^{o p t}$ given by:

$$
\frac{T_{C}^{o p t}}{\tau_{X}}=\left(\frac{\tau_{X}}{\tau_{B X}}-1\right)^{-1} \ln \left(\frac{\tau_{X}}{\tau_{B X}}\right)
$$

The $\mathrm{X}$ and BX quantum yields are related to their radiative lifetimes by:

$$
\frac{Q Y_{B X}}{Q Y_{X}}=\beta \frac{\tau_{B X}}{\tau_{X}}
$$

where $\beta$ is a scaling factor which in this case is determined merely by the simple statistical scaling of the optical recombination pathways as a function of the number of excitons, ${ }^{18,42}$ i.e. $\beta=4$. This is shown schematically in Fig. 1a for the $\mathrm{X}$ and BX states. As compared 
to a standalone NQD (Fig. 1b) the efficiency of TIMED increases with QY ${ }_{B X}$ (Fig. 2d) illustrating the viability of this technique for high two-photon emission probabilities. Furthermore TIMED is a passive scheme requiring no active feedback from the idler port to the switch, which is advantageous due to the simple architecture of the optical circuit. Using the above value of $\beta$ and the optimized $T_{C}^{o p t}$ yields a pure source with $S=1$ with a maximum theoretical efficiency $\eta \simeq 0.63$ (again taking $Q Y_{X}=Q Y_{B X}=1$, see Fig. 2c), already better than the simple beam-splitter technique (which can only reach $\eta=0.5$ even in an

ideal system). For currently realized $Q Y$ values $\left(Q Y_{X}=0.61, Q Y_{B X}=0.7\right)^{18}$ we expect a theoretical efficiency of $26 \%$, compared to $20 \%$ for the beam-splitter technique. This is a significant improvement, yet even better results can be achieved, with an active heralding technique, as is shown next.

\section{Active Heralding:}

This maximum efficiency of TIMED is a limitation of passive heralding techniques. To overcome this limitation in $\eta$ without sacrificing purity a more sophisticated solution, the Active Switching Heralded (ASH) technique is suggested (see Fig.2b). In this active switching scheme the switch is open to the idler port from the beginning of the excitation cycle until a photon detection has occurred. This detection signal would be forwarded to the switch causing it to route the photons to the signal port until the end of the cycle. Realistically, there is a finite time between the arrival of the idler photon to the detector and the switching event which we call the response time $\left(T_{R}\right)$ of the system. This response time takes into account the rise times of the detector and switch and any optical or electronic delays in the system. The heralding efficiency as a function of the response time can be expressed as: ${ }^{40}$

$$
\eta_{A S H}=\alpha^{2} Q Y_{X} Q Y_{B X} \exp \left(-T_{R} / \tau_{X}\right)
$$



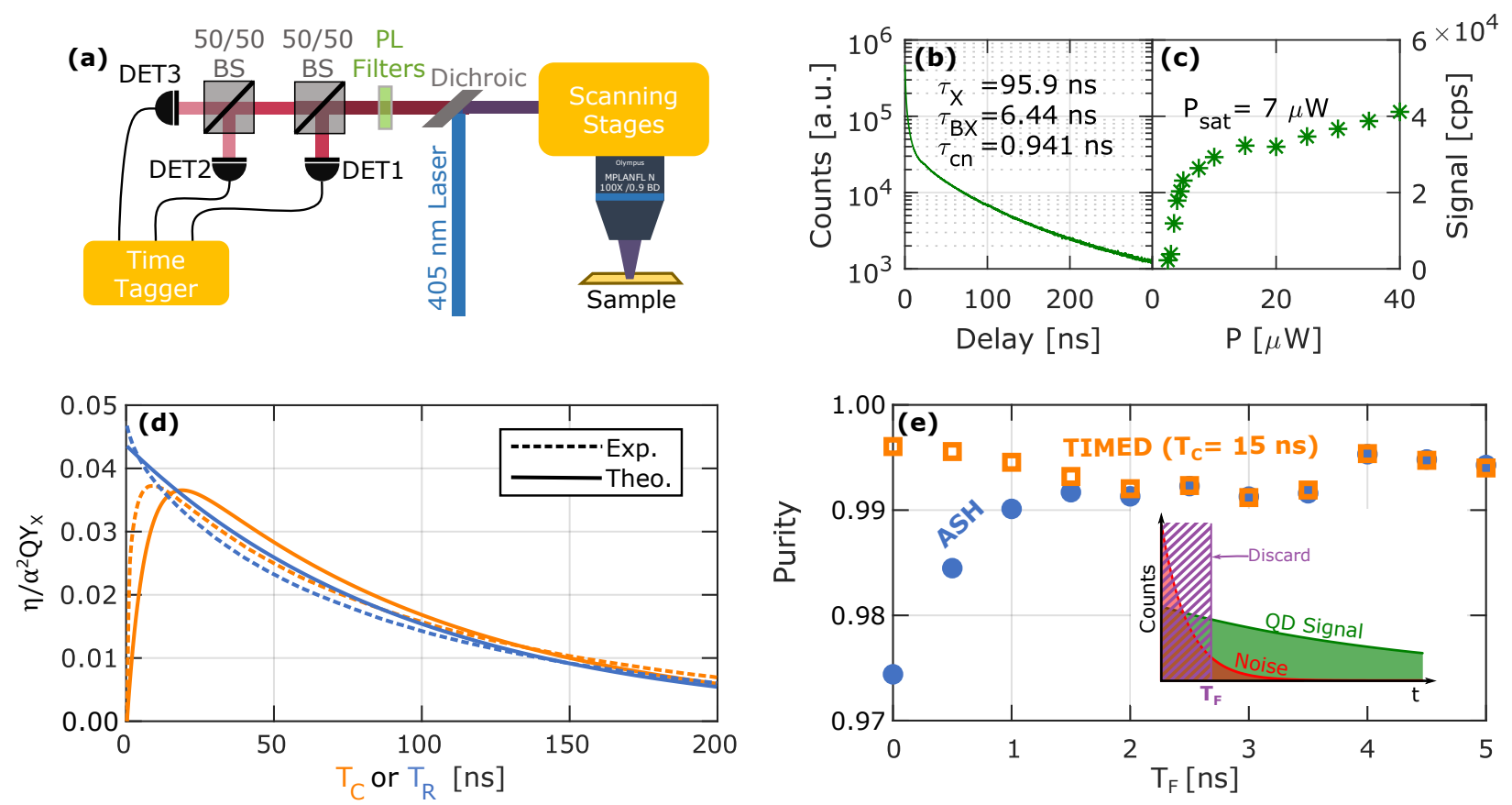

Figure 3: (a) Illustration of the experimental setup used to emulate the TIMED and ASH schemes. (b) Lifetime measurement and (c) power saturation curve for the QD studied in this work.(d) Experimental results (dotted lines) for the efficiency (in units of $\alpha^{2} Q Y_{X}$ in the TIMED and ASH schemes compared to the predicted values (solid lines). (e) The single photon purity as a function of the filtering time which is used to filter out short-time correlated noise illustrated in the inset.

Notably, it can be seen (Fig.2c) that in the ASH scheme, the maximal $\eta$ for the theoretically ideal case $Q Y_{X}=Q Y_{B X}=1$ exponentially approaches $100 \%$ (or $43 \%$ for the case $Q Y_{X}=$ $\left.0.61, Q Y_{B X}=0.7^{18}\right)$, as $T_{R}$ becomes smaller than $\tau_{X}$. Therefore implementing ASH with very short response gates should approach the ideal SPS scenario.

\section{Experimental Emulation:}

Rather than physically implementing the setups for the TIMED and ASH protocols, which require building a dedicated setup for each, we emulate the switching on the time-tagged data obtained from unified experimental setup shown in Fig. 3a (for details regarding the experimental technique and analysis methods refer to the supplementary material). In such a way we can compare the performance of multiple schemes for the same NQD. Fig. 3b 
displays the lifetime measurement for the NQD under investigation, which was fitted to a three exponential fit yielding the $\mathrm{X}$ and BX lifetimes, and a significant short lifetime component which we attribute to a correlated laser-induced noise (hereinafter referred to as correlated noise). Fig. 3c displays the saturation curve for the same NQD indicating that at the maximum power used in this experiment we are pumping well above saturation. This is done to ensure a maximal occupation of the BX states. Using the measured lifetimes and Eq. 2 we find that the ratio between the quantum yields is: $Q Y_{B X} / Q Y_{X}=0.269$. With this ratio, the one photon probability, and our calibrated system collection efficiency $(\alpha=0.088)$ we find the value of each of the quantum yields separately to be: $Q Y_{X}=0.1729$ and $Q Y_{B X}=0.0465 .{ }^{40}$ Prior to any purification protocol we measured the photon purity to be only $S \approx 0.9$ for this NQD. ${ }^{40}$

Fig. 3d displays the experimental efficiency for both TIMED and ASH compared to the theoretically expected values (Eqs. 1 and 3) as a function of $T_{C}$ or $T_{R}$ respectively. We find a good agreement between the experimental results and the expected values for both schemes, and the small shift in the position of maximum of the experimental $\eta_{T I M E D}$ to a lower $T_{C}$ is attributed to the effect of the correlated noise. We find experimentally that the maximal efficiency for the ASH scheme is $\eta_{A S H}=0.0468 \alpha^{2} Q Y_{X}$, which is in agreement with the experimentally extracted value $Q Y_{B X}=0.0465$. Comparing this to Eq. 3 with $T_{R}=0$ indicates that indeed this efficiency is only limited by the quantum yields of the $\mathrm{X}$ and BX, as is theoretically predicted.

Going to the source purity, theoretically, if the NQD cannot emit more than two-photons per excitation pulse, we expect a unity purity, i.e. $S=1$. Experimentally we expect that in this case the sole source reducing $S$ in the signal port is from noise. To check this point we show the purity, $S$, of both schemes as a function of the filtering time $T_{F}$ after the excitation pulse, where all data with $t<T_{F}$ is ignored. This is done to minimize the contribution of short lifetime laser-induced correlated noise. For $T_{F}>1 \mathrm{~ns}, S$ reaches a maximum of around 0.995, after filtering out nearly all correlated noise. We attribute this limit to uncorrelated 
noise rather to inherent multi-photon $(>2)$ emission events from the NQD. ${ }^{40}$

\section{Discussion and Outlook:}
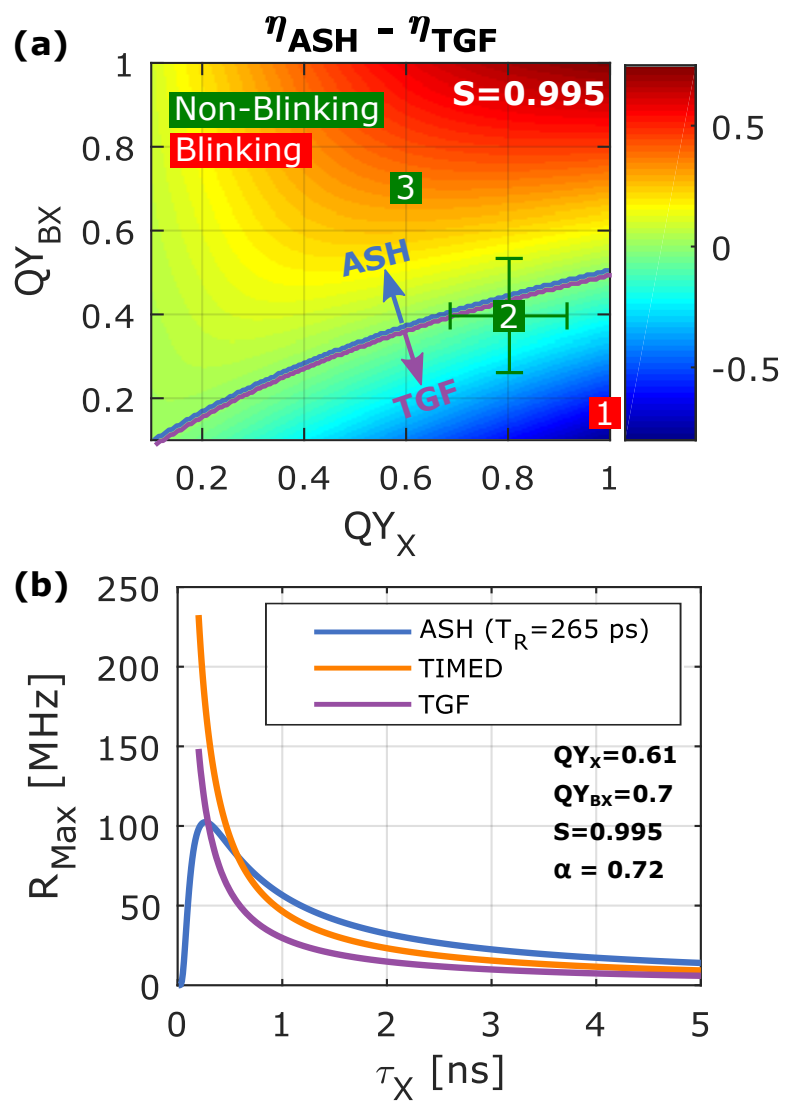

Figure 4: (a) Difference between the efficiency of the ASH and TGF scheme displaying the regions where each technique is superior. The overlaying points are measured values of the $\mathrm{QY}_{X}$ and $\mathrm{QY}_{B X}$ for: (1) Standalone CdSe/CdZnS NQD ${ }^{43}$, (2) CdSe/ 16 monolayer CdS gQD coupled to plasmonic nanocone, ${ }^{18}$ and (3) CdSe/ 19 monolayer CdS standalone gQD. ${ }^{44}$ (b) The single photon rate of ASH, TIMED and TGF as a function of the exciton lifetime for $Q Y_{X}=0.61, Q Y_{B X}=0.7, S=0.995, \alpha=0.72$ and assuming an excitation rate equal to $3 / \tau_{X}$.

As can be seen from the model and the emulation experiments, the heralded purification of single photons from BX emission in NQDs can significantly increase their single photon purity without compromising their efficiencies, which can make such NQD sources (or other sources that have high quantum yield for two-photon emission), good candidates for highquality room-temperature SPSs. To demonstrate the significant superiority of the heralded 
techniques over TGF we plot in Fig. 4a the difference in the efficiency between the ASH and TGF techniques for a specific chosen purity $(\mathrm{S}=0.995)$. This displays that it is not necessary to have very high QYs for the ASH technique to outperform the TGF technique. In fact, currently available NQD systems already are in the regime where ASH is better. Importantly, as the quality of these QDs improve, and as larger and larger Purcell factors are achieved to increase their single photon brightness, it is expected that the values of the QYs would increase making these techniques even more advantageous.

In order to assert the applicability of our techniques and to make a quantitative comparison with other state-of-the-art devices we calculate the expected performance of our techniques based on a model system that was previously introduced, namely an NQD coupled to the near field of a plasmonic nanocone. In Matsuzaki et al. ${ }^{18}$ it has been shown experimentally that this coupling induces a very large Purcell effect, leading to a significant reduction in the radiative lifetimes of both the $\mathrm{X}$ and the $\mathrm{BX}$, and in a very large increase of $Q Y_{B X}$ yielding: $Q Y_{X}=0.61, Q Y_{B X}=0.7, \tau_{X}=1.6 \mathrm{~ns}$, and $\tau_{B X}=0.5 \mathrm{~ns}$. This makes the coupled NQD a rather good bi-photon emitter. In addition we assume a realistic photon collection efficiency of $0.8^{45}$ and a detection efficiency of $90 \%^{46,47}$ (i.e. $\alpha=0.72$ ).

One particularly important point to consider is that for ASH the response time should be fast compared to the $\mathrm{X}$ lifetime. In the supplementary information ${ }^{40}$ we show that an onchip implementation of ASH could realistically have response times of about 265 ps. In the model system suggested in the previous paragraph this would correspond to $T_{R} / \tau_{X} \approx 0.17$ and $\eta_{A S H}=0.19$ compared to $\eta_{T I M E D}=0.15$ and $\eta_{T G F}=0.089$. Under such efficiencies the single photon rate can be estimated to be $R_{A S H}=38 \mathrm{MHz}, R_{T I M E D}=30 \mathrm{MHz}$, and $R_{T G F}=18 \mathrm{MHz}$ under pulsed excitation at a rate of $200 \mathrm{MHz}\left(1 / 3 \tau_{X}\right)$. This is another confirmation that under realistic conditions both heralded techniques can outperform TGF. Nonetheless it is clear that $T_{R}$ imposes a limitation on the maximum $\mathrm{X}$ decay rate that can be achieved before TIMED and TGF outperform ASH. We analyze this situation in Fig.4b where we vary $\tau_{X}$ while holding all other parameters constant as in the suggested 
model system above. As expected at low $\tau_{X}$ ASH suffers however TIMED (TGF) only has better performance for $\tau_{X}<0.6(0.3)$ ns. These values of single photon purity and rate also compare well with state-of-the-art parametric heralded sources, ${ }^{6,7,48}$ and cryogenic-operated self-assembled $\mathrm{QD}^{8,9}$ sources. We note that in contrast to cryogenic QD sources, photons emitted from room-temperature sources are distinguishable in nature. However several recent reports have addressed this issue by proposing cavity enhanced techniques to increase the indistinguishablity of room-temperature emitters. ${ }^{49-51}$

One interesting application, even under limited indistinguishability, is quantum key distribution (QKD) for which true room-temperature SPSs are desired. ${ }^{52}$ In the quantum metrology realm one may devise using these highly intensity-squeezed sources to conduct weak absorption measurements on highly sensitive samples or for calibrating photodetectors with high precision well beyond the shot-noise limit. ${ }^{1}$ Eventually such sources may become the

ultimate standard for intensity measurements in what is known as the quantum candela. ${ }^{53}$ Furthermore the heralding nature of the schemes proposed in this work open up the possibility of multiplexing a few NQDs together to overcome any inefficiencies that may be caused due to inadequate quantum yields or collection efficiencies, thus increasing the attainable single photon rates. ${ }^{40}$

In summary, we show both theoretically and by experimental emulation the ability to break the trade-off between stability, efficiency and single photon purity in statistical twophoton emitters by temporal heralding schemes, allowing to achieve near-unity efficiency and single photon purity simultaneously.

\section{Acknowledgement}

This work was performed, in parts under the financial support from: The Einstein Foundation Berlin; The U.S. Department of Energy: Office of Basic Energy Sciences - Division of Materials Sciences and Engineering; User Project 2017BU0062 at the Center for Integrated 
Nanotechnologies, an U.S. Department of Energy (DOE) Office of Science User Facility operated for the DOE by Los Alamos National Laboratory (LANL) (Contract DE-AC5206NA25396) and Sandia National Laboratories (Contract DE-NA-0003525). J.H. and S.M. acknowledge partial support from the LANL Laboratory Directed Research and Development funds.

\section{Supporting Information Available}

The following files are available free of charge.

The supplementary information contains the theoretical derivations for the equations shown in the text in addition to experimental and analysis details.

\section{References}

(1) Lounis, B.; Orrit, M. Reports on Progress in Physics 2005, 68, 1129-1179.

(2) Eisaman, M. D.; Fan, J.; Migdall, A.; Polyakov, S. V. Review of Scientific Instruments 2011, 82, 071101.

(3) Aharonovich, I.; Englund, D.; Toth, M. Nature Photonics 2016, 10, 631-641.

(4) Paul G. Kwiat, Physical Review Letters 1995,

(5) Yan, Z.; Duan, Y.; Helt, L. G.; Ams, M.; Withford, M. J.; Steel, M. J. Applied Physics Letters 2015,

(6) Ngah, L. A.; Alibart, O.; Labonté, L.; D'Auria, V.; Tanzilli, S. Ultra-fast heralded single photon source based on telecom technology. 2015.

(7) Joshi, C.; Farsi, A.; Clemmen, S.; Ramelow, S.; Gaeta, A. L. Nature Communications $\mathbf{2 0 1 8}, 9,847$. 
(8) Ding, X.; He, Y.; Duan, Z.-C.; Gregersen, N.; Chen, M.-C.; Unsleber, S.; Maier, S.; Schneider, C.; Kamp, M.; Höfling, S.; Lu, C.-Y.; Pan, J.-W. Physical Review Letters 2016, 116, 020401.

(9) Somaschi, N. et al. Nat. Photon. 2016, 10, 340-345.

(10) Schlehahn, A.; Thoma, A.; Munnelly, P.; Kamp, M.; Höfling, S.; Heindel, T.; Schneider, C.; Reitzenstein, S. APL Photonics 2016, 1, 011301.

(11) Daveau, R. S.; Balram, K. C.; Pregnolato, T.; Liu, J.; Lee, E. H.; Song, J. D.; Verma, V.; Mirin, R.; Nam, S. W.; Midolo, L.; Stobbe, S.; Srinivasan, K.; Lodahl, P. Optica 2017, 4, 178 .

(12) Liu, F.; Brash, A. J.; O’Hara, J.; Martins, L. M. P. P.; Phillips, C. L.; Coles, R. J.; Royall, B.; Clarke, E.; Bentham, C.; Prtljaga, N.; Itskevich, I. E.; Wilson, L. R.; Skolnick, M. S.; Fox, A. M. 2017,

(13) Lounis, B.; Bechtel, H.; Gerion, D.; Alivisatos, P.; Moerner, W.

(14) Michler, P.; Imamoğlu, A.; Mason, M. D.; Carson, P. J.; Strouse, G. F.; Buratto, S. K. Nature 2000, 406, 968-970.

(15) Chen, Y.; Vela, J.; Htoon, H.; Casson, J. L.; Werder, D. J.; Bussian, D. A.; Klimov, V. I.; Hollingsworth, J. A. Journal of the American Chemical Society 2008, 130, 5026-5027.

(16) Bidault, S.; Devilez, A.; Maillard, V.; Lermusiaux, L.; Guigner, J.-M.; Bonod, N.; Wenger, J. ACS Nano 2016, 10, 4806-4815.

(17) Hoang, T. B.; Akselrod, G. M.; Mikkelsen, M. H. Nano Letters 2016, 16, 270-275.

(18) Matsuzaki, K.; Vassant, S.; Liu, H.-W.; Dutschke, A.; Hoffmann, B.; Chen, X.; Christiansen, S.; Buck, M. R.; Hollingsworth, J. A.; Götzinger, S.; Sandoghdar, V. Scientific Reports 2017, 7, 42307. 
(19) Aharonovich, I.; Neu, E. Advanced Optical Materials 2014, 2, 911-928.

(20) Schröder, T.; Mouradian, S. L.; Zheng, J.; Trusheim, M. E.; Walsh, M.; Chen, E. H.; Li, L.; Bayn, I.; Englund, D. Journal of the Optical Society of America B 2016, 33, B65.

(21) Castelletto, S.; Johnson, B. C.; Ivády, V.; Stavrias, N.; Umeda, T.; Gali, A.; Ohshima, T. Nature Materials 2014, 13, 151-156.

(22) He, X.; Hartmann, N. F.; Ma, X.; Kim, Y.; Ihly, R.; Blackburn, J. L.; Gao, W.; Kono, J.; Yomogida, Y.; Hirano, A.; Tanaka, T.; Kataura, H.; Htoon, H.; Doorn, S. K. Nature Photonics 2017, 11, 577-582.

(23) Ishii, A.; Uda, T.; Kato, Y. Physical Review Applied 2017, 8, 054039.

(24) Chakraborty, C.; Kinnischtzke, L.; Goodfellow, K. M.; Beams, R.; Vamivakas, A. N. Nature Nanotechnology 2015, 10, 507-511.

(25) Grosso, G.; Moon, H.; Lienhard, B.; Ali, S.; Efetov, D. K.; Furchi, M. M.; JarilloHerrero, P.; Ford, M. J.; Aharonovich, I.; Englund, D. Nature Communications 2017, 8,705 .

(26) Arita, M.; Le Roux, F.; Holmes, M. J.; Kako, S.; Arakawa, Y. Nano Letters 2017, 17, 2902-2907.

(27) Efros, A. L.; Nesbitt, D. J. Nature Nanotechnology 2016, 11, 661-671.

(28) Park, Y.-S.; Ghosh, Y.; Xu, P.; Mack, N. H.; Wang, H.-L.; Hollingsworth, J. A.; Htoon, H. The Journal of Physical Chemistry Letters 2013, 4, 1465-1470.

(29) Wang, F.; Karan, N. S.; Nguyen, H. M.; Ghosh, Y.; Sheehan, C. J.; Hollingsworth, J. A.; Htoon, H. Nanoscale 2015, 7, 9387-9393.

(30) Dey, S.; Zhao, J. The Journal of Physical Chemistry Letters 2016, 7, 2921-2929. 
(31) Mangum, B. D.; Sampat, S.; Ghosh, Y.; Hollingsworth, J. A.; Htoon, H.; Malko, A. V. Nanoscale 2014, 6, 3712 .

(32) Brokmann, X.; Messin, G.; Desbiolles, P.; Giacobino, E.; Dahan, M.; Hermier, J. P. New Journal of Physics 2004, 6, 99-99.

(33) Livneh, N.; Harats, M. G.; Yochelis, S.; Paltiel, Y.; Rapaport, R. ACS Photonics 2015, 2, 1669-1674.

(34) Livneh, N.; Harats, M. G.; Istrati, D.; Eisenberg, H. S.; Rapaport, R. Nano Letters 2016, 16, 2527-2532.

(35) Lin, X.; Dai, X.; Pu, C.; Deng, Y.; Niu, Y.; Tong, L.; Fang, W.; Jin, Y.; Peng, X. Nature Communications 2017, 8, 1132.

(36) Chandrasekaran, V.; Tessier, M. D.; Dupont, D.; Geiregat, P.; Hens, Z.; Brainis, E. Nano Letters 2017, 17, 6104-6109.

(37) Htoon, H.; Malko, A. V.; Bussian, D.; Vela, J.; Chen, Y.; Hollingsworth, J. A.; Klimov, V. I. Nano Letters 2010, 10, 2401-2407.

(38) Mangum, B. D.; Ghosh, Y.; Hollingsworth, J. A.; Htoon, H. Optics express 2013, 21, 7419-26.

(39) Feng, S.-W.; Cheng, C.-Y.; Wei, C.-Y.; Yang, J.-H.; Chen, Y.-R.; Chuang, Y.-W.; Fan, Y.-H.; Chuu, C.-S. Physical Review Letters 2017, 119, 143601.

(40) see supplementary information for further details.

(41) In this analysis we assume that the NQD is pumped well above saturation with a short pulse so as to prepare the NQD in the BX state at the beginning of each excitation cycle.

(42) Park, Y.-S.; Bae, W. K.; Pietryga, J. M.; Klimov, V. I. ACS Nano 2014, 8, 7288-7296. 
(43) Nair, G.; Zhao, J.; Bawendi, M. G. Nano Letters 2011, 11, 1136-1140.

(44) Park, Y.-S.; Malko, A. V.; Vela, J.; Chen, Y.; Ghosh, Y.; García-Santamaría, F.; Hollingsworth, J. A.; Klimov, V. I.; Htoon, H. Physical Review Letters 2011, 106, 187401.

(45) Abudayyeh, H.; Rapaport, R. Quantum Science and Technology 2017,

(46) Ma, J.; Zhou, M.; Yu, Z.; Jiang, X.; Huo, Y.; Zang, K.; Zhang, J.; Harris, J. S.; Jin, G.; Zhang, Q.; Pan, J.-W. Optica 2015, 2, 974.

(47) Zang, K.; Jiang, X.; Huo, Y.; Ding, X.; Morea, M.; Chen, X.; Lu, C.-Y.; Ma, J.; Zhou, M.; Xia, Z.; Yu, Z.; Kamins, T. I.; Zhang, Q.; Harris, J. S. Nature Communications 2017, 8, 628 .

(48) Wang, X.-L. et al. Physical Review Letters 2016, 117, 210502.

(49) Grange, T.; Hornecker, G.; Hunger, D.; Poizat, J. P.; Gérard, J. M.; Senellart, P.; Auffeves, A. Physical Review Letters 2015, 114, 1-5.

(50) Wein, S.; Lauk, N.; Ghobadi, R.; Simon, C. Physical Review B 2018, 97, 205418.

(51) Choi, H.; Zhu, D.; Englund, D. Highly Indistinguishable Room Temperature Single Photon Sources with Quantum Emitters in Bad Cavity Regime. Conference on Lasers and Electro-Optics. Washington, D.C., 2018; p FM1H.6.

(52) Takemoto, K.; Nambu, Y.; Miyazawa, T.; Sakuma, Y.; Yamamoto, T.; Yorozu, S.; Arakawa, Y. Scientific Reports 2015, 5, 14383.

(53) Cheung, J. Y.; Chunnilall, C. J.; Woolliams, E. R.; Fox, N. P.; Mountford, J. R.; Wang, J.; Thomas, P. J. Journal of Modern Optics 2007, 54, 373-396. 


\title{
Supplementary Information \\ Purification of single photons by temporal heralding of quantum dot sources
}

\author{
Hamza Abudayyeh, Boaz Lubotzky, and Ronen Rapaport* \\ Racah Institute for Physics and Center for Nanoscience and Nanotechnology, \\ The Hebrew University of Jerusalem, Jerusalem 9190401, Israel \\ Somak Majumder and Jennifer A. Hollingsworth \\ Materials Physics \&6 Applications Division: Center for Integrated Nanotechnologies, \\ Los Alamos National Laboratory, Los Alamos, New Mexico 87545, United States
}

* ronenr@phys.huji.ac.il 


\section{THEORETICAL DERIVATION OF HERALDING PARAMETERS} $\Gamma_{i}$ :

The quantum yield of an emission process $i$ is given by the ratio of the radiative decay rate $\Gamma_{i}^{r}$ to total decay rate

$$
Q Y_{i}=\frac{\Gamma_{i}^{r}}{\Gamma_{i}}
$$

For a biexciton-exciton emission cascade simple statistical scaling implies that the radiative decay rate of the biexciton should be 4 times that of the exciton. In reality other factors play a role such as the degree of confinement of the electrons and holes to the core of a core/shell quantum dot for example. Therefore in general this scaling factor will be called $\beta$, i.e. $\Gamma_{B X}^{r}=\beta \Gamma_{X}^{r}$ and thus:

$$
\begin{aligned}
\frac{Q Y_{B X}}{Q Y_{X}} & =\frac{\Gamma_{B X}^{r} \Gamma_{X}}{\Gamma_{B X} \Gamma_{X}^{r}} \\
& =\frac{\beta \Gamma_{X}^{r} \Gamma_{X}}{\Gamma_{B X} \Gamma_{X}^{r}} \\
& =\beta \frac{\Gamma_{X}}{\Gamma_{B X}}
\end{aligned}
$$

Therefore if the $\beta$-scaling factor is known a simple lifetime measurement can be used to find the ratio of the biexciton to exciton quantum yield.

We will assume that our nanocrystal quantum dot can emit at most two photons based on the biexciton-exciton cascade and that it is being pumped well above saturation. The probability that a biexciton photon is emitted $T_{B X}$ after the laser pulse is given by:

$$
p\left(T_{B X}\right)=\alpha Q Y_{B X} \Gamma_{B X} e^{-\Gamma_{B X} T_{B X}}
$$

whereas the exciton emission time $\left(T_{X}\right)$ is conditioned on the emission time of the biexciton as follows:

$$
p\left(T_{x} \mid T_{B X}\right)=\alpha Q Y_{X} \Gamma_{X} e^{-\Gamma_{X}\left(T_{X}-T_{B X}\right)}
$$

where:

$\alpha: \quad$ overall detection efficiency of the system

$Q Y_{i}: \quad$ quantum yield of the $i^{\text {th }}$ emission process

$p\left(T_{B X}\right): \quad$ probability of biexciton event at local time $T_{B X}$

$p\left(T_{x} \mid T_{B X}\right)$ : conditional probability of exciton event at local time $T_{X}$ given that a biexciton event was detected at $T_{B X}$

Therefore for a standalone NQD the probability to emit one and two photons ( $P_{1}$ and $P_{2}$ respectively) are:

$$
\begin{gathered}
P_{1}=\alpha Q Y_{X}+\alpha Q Y_{B X}-2 \alpha^{2} Q Y_{X} Q Y_{B X} \\
P_{2}=\alpha^{2} Q Y_{X} Q Y_{B X}
\end{gathered}
$$

\section{I.A. Fixed gate techniques}

If we assume that a fixed time gate is applied as in the case of TGF and TIMED than we can ask what is the probability that a photon will take a certain route. For example what is the probability that one photon will arrive within $T$ from the laser pulse and the other photon will arrive after $T$. This probability is given by the following integral:

$$
\begin{aligned}
\mathscr{P}_{1}(T) & =\alpha^{2} Q Y_{X} Q Y_{B X} \int_{0}^{T} d T_{B X} \int_{T}^{\infty} d T_{X} p\left(T_{B X}\right) p\left(T_{x} \mid T_{B X}\right) \\
& =\alpha^{2} Q Y_{X} Q Y_{B X} \frac{\Gamma_{B X}}{\Gamma_{X}-\Gamma_{B X}} \exp \left(-\Gamma_{X} T\right)\left\{1-\exp \left(-\left(\Gamma_{B X}-\Gamma_{X}\right) T\right)\right\}
\end{aligned}
$$


TABLE S1. Possible events resulting from an emitter that has a maximum of a two photon cascade by applying a fixed time gate

\begin{tabular}{|c|c|c|c|}
\hline \multicolumn{4}{|c|}{ Condition on BX Condition on X Prob. of Process } \\
\hline 1. & $T_{B X} \leq T$ & $T_{X} \geq T$ & $\mathscr{P}_{1}(T)=\alpha^{2} Q Y_{X} Q Y_{B X} \frac{\Gamma_{B X}}{\Gamma_{B X}-\Gamma_{X}}\left\{\exp \left(-\Gamma_{X} T\right)-\exp \left(-\Gamma_{B X} T\right)\right\}$ \\
\hline 2. & $T_{B X} \leq T$ & $T_{X} \leq T$ & $\mathscr{P}_{2}(T)=\alpha^{2} Q Y_{B X} Q Y_{x}\left\{1-\frac{\Gamma_{B X}}{\Gamma_{B X}-\Gamma_{X}} \exp \left(-\Gamma_{X} T\right)+\frac{\Gamma_{X}}{\Gamma_{B X}-\Gamma_{X}} \exp \left(-\Gamma_{B X} T\right)\right\}$ \\
\hline 3. & $T_{B X} \geq T$ & $T_{X} \geq T$ & $\mathscr{P}_{3}(T)=\alpha^{2} Q Y_{B X} Q Y_{x} \exp \left(-\Gamma_{B X} T\right)$ \\
\hline 4. & $T_{B X} \leq T$ & - & $\mathscr{P}_{4}(T)=\alpha Q Y_{B X}\left(1-\alpha Q Y_{X}\right)\left\{1-\exp \left(-\Gamma_{B X} T\right)\right\}$ \\
\hline 5. & $T_{B X} \geq T$ & - & $\mathscr{P}_{5}(T)=\alpha Q Y_{B X}\left(1-\alpha Q Y_{X}\right) \exp \left(-\Gamma_{B X} T\right)$ \\
\hline 6. & - & $T_{X} \leq T$ & $\mathscr{P}_{6}(T)=\alpha Q Y_{X}\left(1-\alpha Q Y_{B X}\right)\left\{1-\exp \left(-\Gamma_{X} T\right)\right\}$ \\
\hline 7. & - & $T_{X} \geq T$ & $\mathscr{P}_{7}(T)=\alpha Q Y_{X}\left(1-\alpha Q Y_{B X}\right) \exp \left(-\Gamma_{X} T\right)$ \\
\hline 8. & - & - & $\mathscr{P}_{8}=\left(1-\alpha Q Y_{B X}\right)\left(1-\alpha Q Y_{X}\right)$ \\
\hline
\end{tabular}

On the other hand the probability that both will arrive prior to $T\left(\mathscr{P}_{2}(T)\right)$ or after $T\left(\mathscr{P}_{3}(T)\right)$ are given by the following integrals:

$$
\begin{aligned}
& \mathscr{P}_{2}(T)=\alpha^{2} Q Y_{X} Q Y_{B X} \int_{0}^{T} d T_{B X} \int_{T_{B X}}^{T} d T_{X} p\left(T_{B X}\right) p\left(T_{x} \mid T_{B X}\right) \\
& \mathscr{P}_{3}(T)=\alpha^{2} Q Y_{X} Q Y_{B X} \int_{T}^{\infty} d T_{B X} \int_{T_{B X}}^{\infty} d T_{X} p\left(T_{B X}\right) p\left(T_{x} \mid T_{B X}\right)
\end{aligned}
$$

These events in addition to all other possibilities are summarized in table S1.

In Time Gated Filtering (TGF) shown in figure S1a the switch is open to an optical dump from the beginning of each pulse up to a filtering time $T_{F}$ after which the photons are routed to the signal port. In such a manner the short lifetime components of an optical signal can be filtered out. This can be used to filter out the biexciton emission where the the efficiency of the source would be the probability of only one photon events after a time gate of $T_{F}$ namely:

$$
\eta_{T G F}=\mathscr{P}_{1}\left(T_{F}\right)+\mathscr{P}_{5}\left(T_{F}\right)+\mathscr{P}_{7}\left(T_{F}\right)
$$

where as the purity is the same quantity normalized by the probability of obtaining one or two photons after $T_{F}$ which is given by:

$$
S_{T G F}=\frac{\mathscr{P}_{1}\left(T_{F}\right)+\mathscr{P}_{5}\left(T_{F}\right)+\mathscr{P}_{7}\left(T_{F}\right)}{\mathscr{P}_{1}\left(T_{F}\right)+\mathscr{P}_{3}\left(T_{F}\right)+\mathscr{P}_{5}\left(T_{F}\right)+\mathscr{P}_{7}\left(T_{F}\right)}
$$

This case of TGF with $\mathrm{QY}_{X}=0.6$ and $\mathrm{QY}_{B X}=0.71[1]$ is plotted in figure S1b where the trade-off between purity and efficiency in terms of $T_{F}$ is clear.

For the special case of unity collection efficiency and unity quantum yields the expressions reduce to:

$$
\begin{gathered}
\eta_{T G F}^{\text {ideal }}=\frac{\Gamma_{B X}}{\Gamma_{B X}-\Gamma_{X}}\left\{\exp \left(-\Gamma_{X} T_{F}\right)-\exp \left(-\Gamma_{B X} T_{F}\right)\right\} \\
S_{T G F}^{i d e a l}=\frac{\Gamma_{B X}\left\{\exp \left(-\Gamma_{X} T_{F}\right)-\exp \left(-\Gamma_{B X} T_{F}\right)\right\}}{\Gamma_{B X} \exp \left(-\Gamma_{X} T_{F}\right)-\Gamma_{X} \exp \left(-\Gamma_{B X} T_{F}\right)}
\end{gathered}
$$

On the other hand for the TIMe resolved heraldED (TIMED) scheme only the first process constitutes a successful heralding event namely:

$$
\eta_{T I M E D}=\mathscr{P}_{1}\left(T_{C}\right)=\alpha^{2} Q Y_{X} Q Y_{B X} \frac{\Gamma_{B X}}{\Gamma_{B X}-\Gamma_{X}}\left\{\exp \left(-\Gamma_{X} T_{C}\right)-\exp \left(-\Gamma_{B X} T_{C}\right)\right\}
$$


By using $\tau_{i}=\Gamma_{i}^{-1}$ for $i=X, B X$ we arrive at the equation stated in the main text.

We can choose the optimum cutoff time $T_{c}^{o p t}$ that will maximize $\eta$. This is given in units of the exciton lifetime as:

$$
\Gamma_{x} T_{c}^{o p t}=\left(\frac{\Gamma_{B X}}{\Gamma_{X}}-1\right)^{-1} \ln \left(\frac{\Gamma_{B X}}{\Gamma_{X}}\right)
$$

Using this the optimum efficiency $\eta^{o p t}$ is :

$$
\eta_{T I M E D}^{\text {opt }}=\alpha^{2} Q Y_{X} Q Y_{B X} \frac{\Gamma_{B X} / \Gamma_{X}}{\Gamma_{B X} / \Gamma_{X}-1}\left\{\left(\frac{\Gamma_{B X}}{\Gamma_{X}}\right)^{\frac{-1}{\Gamma_{B X / \Gamma_{X}-1}}}-\left(\frac{\Gamma_{B X}}{\Gamma_{X}}\right)^{\frac{-\Gamma_{B X} / \Gamma_{X}}{\Gamma_{B X} / \Gamma_{X}-1}}\right\}
$$

An important parameter for the case of multiplexed sources is the determinicity defined as the ratio of true heralded events to overall trigger events. This parameter defines the reliability of the trigger signal in heralding a signal photon. In statistics this often called the Positive Predictive Value (PPV) defined as the ratio of true postives to overall postives, but we will stick to the term determinicity for comparasion with non-heralded sources. For the TIMED scheme the determinicity $D$ is given by:

$$
D_{T I M E D}=\frac{\mathscr{P}_{1}\left(T_{C}\right)}{\mathscr{P}_{1}\left(T_{C}\right)+\mathscr{P}_{2}\left(T_{C}\right)+\mathscr{P}_{4}\left(T_{C}\right)+\mathscr{P}_{6}\left(T_{C}\right)}
$$

Again for the idealistic case of unity collection efficiency and quantum yields this reduces to:

$$
D_{\text {TIMED }}^{\text {ideal }}=\frac{\Gamma_{B X}}{\Gamma_{B X}-\Gamma_{X}} \frac{\exp \left(-\Gamma_{X} T_{C}\right)-\exp \left(-\Gamma_{B X} T_{C}\right)}{1-\exp \left(-\Gamma_{B X} T\right)}
$$

\section{I.B. Active Switching Heralded (ASH) scheme}

Due to the active switching the only parameter of importance for this scheme is the resolution time $T_{R}$ of the system. It is the difference in the arrival time between the two photons that needs to be taken into consideration. There are five possible outcomes:

- Two photons with temporal seperation more than $T_{R}$ (successful heralding event) :

$$
\begin{aligned}
\mathscr{P}_{2 S} & =\alpha^{2} Q Y_{X} Q Y_{B X} \int_{0}^{\infty} d T_{B X} \int_{T_{B X}+T_{R}}^{\infty} d T_{X} p\left(T_{B X}\right) p\left(T_{x} \mid T_{B X}\right) \\
& =\alpha^{2} Q Y_{X} Q Y_{B X} \exp \left(-\Gamma_{X} T_{R}\right)
\end{aligned}
$$

- Two photons with temporal seperation arriving within $T_{R}$ from each other:

$$
\begin{aligned}
\mathscr{P}_{2 F} & =\alpha^{2} Q Y_{X} Q Y_{B X} \int_{0}^{\infty} d T_{B X} \int_{T_{B X}}^{T_{B X}+T_{R}} d T_{X} p\left(T_{B X}\right) p\left(T_{x} \mid T_{B X}\right) \\
& =\alpha^{2} Q Y_{X} Q Y_{B X}\left\{1-\exp \left(-\Gamma_{X} T_{R}\right)\right\}
\end{aligned}
$$

- Exciton photon emission only:

$$
\mathscr{P}_{1 X}=\alpha Q Y_{X}\left(1-\alpha Q Y_{B X}\right)
$$

- Biexciton photon emission only:

$$
\mathscr{P}_{1 B X}=\alpha Q Y_{B X}\left(1-\alpha Q Y_{X}\right)
$$




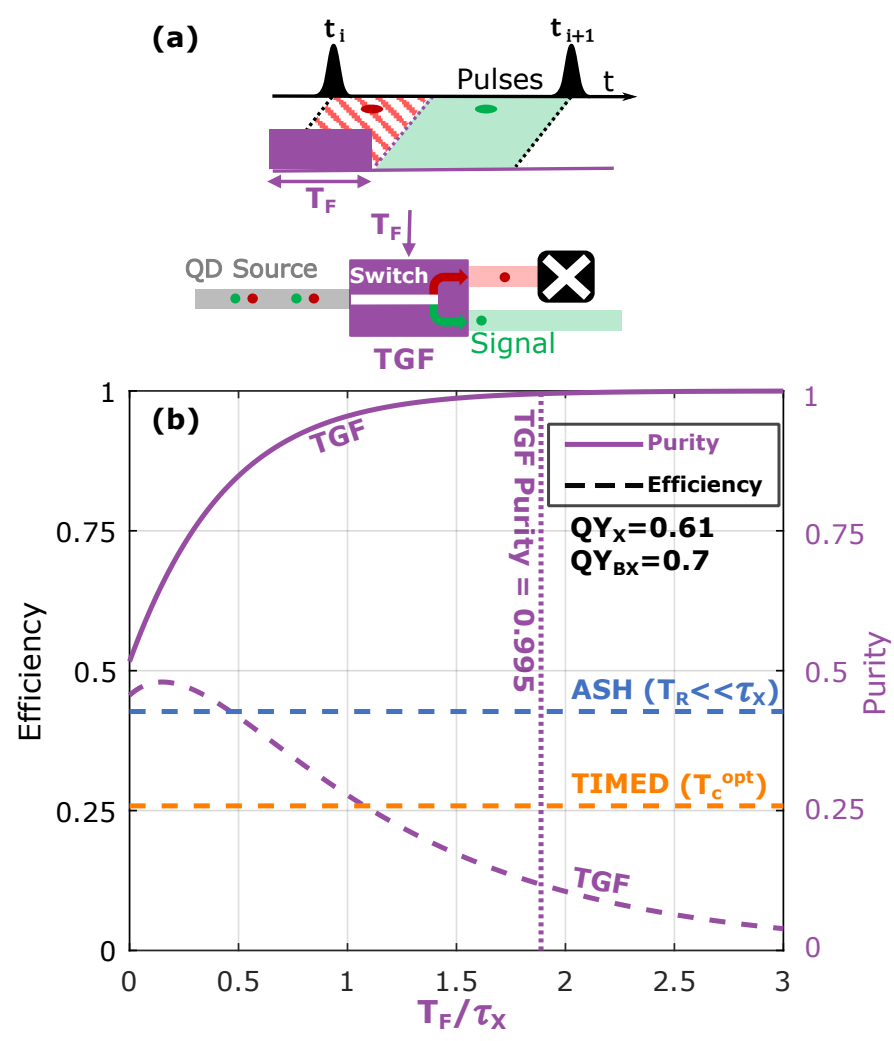

FIG. S1. (a) Schematic of Time-Gated Filtering technique (TGF). (b) The purity (solid line) and efficiency (dashed lines) of the TGF technique for the quantum yields corresponding to a gQD coupled to a nanocone [1]. This is compared to the efficiency of the ASH and TIMED technique

- No photon emission:

$$
\mathscr{P}_{0}=\left(1-\alpha Q Y_{X}\right)\left(1-\alpha Q Y_{B X}\right)
$$

Only the first of these events is a successful heralding event therefore the efficiency is given by:

$$
\eta_{A S H}=\alpha^{2} Q Y_{X} Q Y_{B X} \exp \left(-\Gamma_{X} T_{R}\right)
$$

On the other hand all but the last of these outcomes still will consitute a trigger event therefore the determinicity can be written as:

$$
\begin{aligned}
D_{A S H} & =\frac{\mathscr{P}_{2 S}}{\mathscr{P}_{2 S}+\mathscr{P}_{2 F}+\mathscr{P}_{1 X}+\mathscr{P}_{1 B X}} \\
& =\frac{\alpha^{2} Q Y_{X} Q Y_{B X} \exp \left(-\Gamma_{X} T_{R}\right)}{\alpha Q Y_{X}+\alpha Q Y_{B X}+\alpha^{2} Q Y_{X} Q Y_{B X}}
\end{aligned}
$$

To establish the relevance of these techniques we compare the efficiency of ASH and TIMED to the simpler TGF technique for the case of $Q Y_{X}=0.61$ and $Q Y_{B X}=0.7$ (see main text) [1] in figure S1b. For TGF $T_{F}>1.8$ (indicated by the dotted line) is needed to produce the same purity (0.995) measured in our heralded experiments. For this value of $T_{F}$ it is clear that the efficiency of both ASH and TIMED are superior to TGF.

Figure S2 displays the determinicity as a function of the quantum yield for equal quantum yields (solid line) and for the case where $Q Y_{B X}=0.5 Q Y_{X}$ (dashed line). As can be clearly seen the ASH scheme has a determinicity of unity for unity quantum yields but TIMED reaches to a maximum of around $75 \%$ when the optimum cutoff time is 


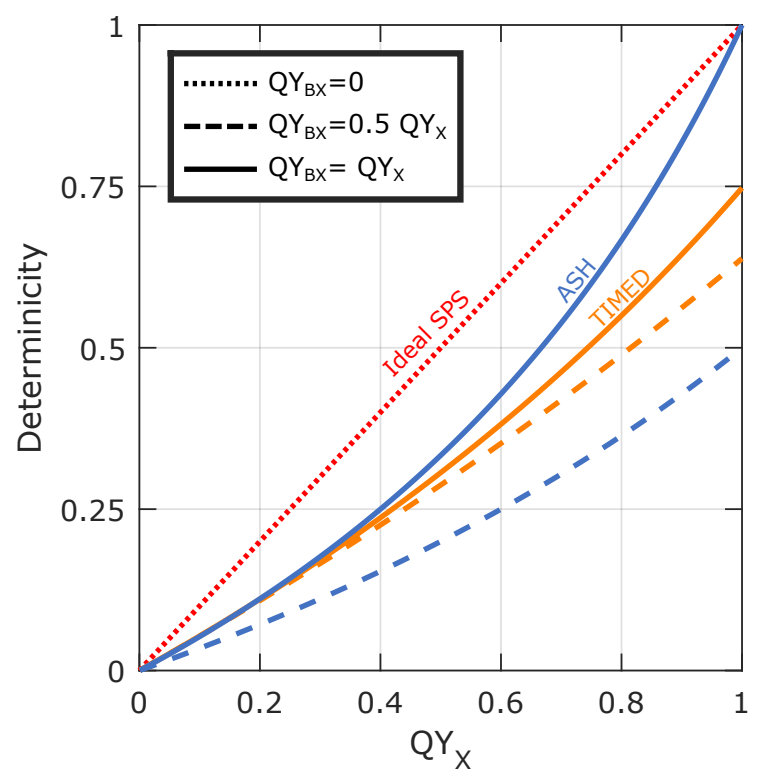

FIG. S2. Determinicity as a function of the quantum yield for ASH $\left(T_{R}<<\tau_{X}\right)$ and TIMED $\left(T_{C}^{o p t}\right)$ as compared to an ideal SPS

used. It should be noted however that this cutoff time was optimized for efficiency not determinicity and in principle one can operate at higher determinicities at the cost of lower efficiency. In the non-ideal case $\left(Q Y_{B X}=0.5 Q Y_{X}\right)$ the TIMED scheme overcomes the ASH scheme since in this case false positives start to appear. ASH is more susceptible to false triggers since it is always open to the idler port until a trigger photon has arrived, whereas the TIMED scheme switches after $T_{C}$ regardless of the presence of a trigger photon or not.

Determinicity is an important parameter for a multiplexed heralded source. This parameter is important because it defines the upper limit for the overall efficiency of the multiplexed source since the optical router will rely on the trigger signal to switch between different components. The schemes proposed in this paper have maximum theoretical determinicities that approach unity for optimized parameters.

\section{EXPERIMENTAL DETAILS}

\section{II.A. Setup and data format}

The measurement is conducted by spin coating a sample of core/thick shell CdSe/CdS nanocrystal quantum dots dispersed in PMMA onto a silicon wafer. The input laser light is a femtosecond $2 \mathrm{MHz} 405 \mathrm{~nm}$ pulsed laser generated by second harmonic generation of a femtosecond Ti:Sapphire laser operating at $810 \mathrm{~nm}$. The sample is scanned using a periscopic system of scanning stages and the laser light is focused onto the sample using a 0.9 NA objective (Olympus MPLFLN100xBD). The emission from a single NQD is collected using the same objective and directed to the collection arm using a 600nm shortpass dichroic mirror. The sample is then imaged using a CMOS camera to verify the excitation of a single NQD. After that the emission is redirected to a set of beam-splitters and single photon avalanche photodiodes (Excelitas SPCM-AQRH-14-FC) as shown in figure 3a in the main text.

The signal from each detector is routed to a different channel on the timetagger device (Swabian TimeTagger 2.0). The output from the timetagger to the computer is a vector containing the arrival times of all the photons within the set exposure time with respect to the beginning of the measurement in addition to a tag labeling which channel this count came from. These arrival times are commonly referred to as global times. Another channel on the time-tagger records the excitation pulse times. By comparing the global time of each count with the nearest preceding laser pulse one can find the local time for the count. A histogram of these local times is what constitutes a lifetime measurement. Therefore at the end of this step we have information about which pulse and what channel the count came from, and its global and local times. This constitutes all the information needed for subsequent analysis steps. 


\section{II.B. Photon number correction}

Due to the deadtime of each detector, counts from the same detector within the same pulse must be neglected. If this was not conducted then a bias would be introduced since two photons arriving with a time separation less than the dead time would not be detected whereas photons separated by more than the deadtime would be. However this does introduce an error in estimating the two and three photon counts due to the finite probability that two or more photons would arrive at the same detector. To correct for this error we must the calculate the probability for the photons to arrive at different detectors and correct the photon counts by dividing by this probability. We have a two beamsplitter scheme the first with a reflectivity $R_{1}$ and the second with a reflectivity $R_{2}$. Assuming two photons were emitted the probability that they would arrive at different detectors is a simple exercise in probabilities and is given by:

$$
P_{\text {diff }}^{(2)}=2 R_{1}\left(1-R_{1}\right)+\left(1-R_{1}\right)^{2}\left[2 R_{2}\left(1-R_{2}\right)\right]
$$

If the pulse contains three photons the probability of them each arriving at a different detector is given by:

$$
P_{\text {diff }}^{(3)}=3 R_{1}\left(1-R_{1}\right)^{2}\left[2 R_{2}\left(1-R_{2}\right)\right]=6 R_{1} R_{2}\left(1-R_{1}\right)^{2}\left(1-R_{2}\right)
$$

Therefore the "true" photon numbers $\left(N_{2}\right.$ and $\left.N_{3}\right)$ are related to the measured ones $\left(N_{2 m}\right.$ and $\left.N_{3 m}\right)$ by:

$$
\begin{aligned}
& N_{2}=\frac{N_{2 m}}{P_{d i f f}^{(2)}} \\
& N_{3}=\frac{N_{3 m}}{P_{d i f f}^{(3)}}
\end{aligned}
$$

In our case $R_{1}=0.4, R_{2}=0.5$ and therefore:

$$
\begin{aligned}
& N_{2}=1.52 N_{2 m} \\
& N_{3}=4.63 N_{3 m}
\end{aligned}
$$

From now on it will be assumed that this correction is conducted whenever there is a two photon or three photon event.

\section{II.C. Analysis Method}

Prior to purification the purity of the QD was calculated by comparing the number of multiphoton $\left(N_{\geq 2}\right)$ events to single photon events $\left(N_{1}\right)$ using this formula:

$$
S=\frac{N_{1}}{N_{1}+N_{2} / \alpha+N_{3} / \alpha^{2}}
$$

Higher order terms were neglected due to the much lower probability of four or more emission events. This also is consistent with the ability to resolve up to three photons in our setup. The factor of $\alpha$ in the denominator is to account for the extra optical loss that a two photon and three photon states encounter.

In the heralded schemes we first filter out all counts having local times less than $T_{F}=300$ ps to reduce the effect of correlated noise. Then all pulses containing two or more photons were chosen for further analysis.

For the TIMED scheme the condition implemented afterwards is that one (or more) photons had a local time less than $T_{C}$ and one (or more) photons had a local time more than $T_{C}$. The number of these events constituted the number of successful heralding events and divided by the overall number of excitation pulses yields the heralding efficiency. This is what is plotted in figure $3 \mathrm{~d}$ in the main text. From these figures we chose the $T_{C}$ that yields the maximum efficiency and for the successful heralding events we count the number of photons in each case that arrive after $T_{C}$ which will be called the signal photons. The number of cases in which we have two signal photons per heralding event $N_{2}$ compared to the number of cases where there is only one $N_{1}$ gives the purity: $S=1-N_{2} / \alpha N_{1}$ 
TABLE S2. System collection efficiency estimation

\begin{tabular}{|c|c|c|}
\hline Component & Method & Efficiency \\
\hline Collection into objective & sim & $39.0 \%$ \\
\hline Objective transmission & meas & $90.0 \%$ \\
\hline 600 nm SP dichroic & meas & $96.6 \%$ \\
\hline $700 \mathrm{~nm}$ SP filter & meas & $88.5 \%$ \\
\hline $550 \mathrm{~nm}$ LP filter & meas & $95.2 \%$ \\
\hline 600 nm LP filter & meas & $84.3 \%$ \\
\hline Beam splitters (2) & meas & $86.3 \%$ \\
\hline Mirrors (6) & meas & $75.9 \%$ \\
\hline Fiber coupling & meas & $80.0 \%$ \\
\hline Detector efficiency & fact & $70.0 \%$ \\
\hline \multicolumn{2}{|c|}{ Total } & $\mathbf{8 . 8 3 \%}$ \\
\hline
\end{tabular}

which can be plotted as a function of the original filtering time $T_{F}$ as in figure $3 \mathrm{e}$ in the main text. The factor of $\alpha$ in the denominator is to account for the extra optical loss that a two photon state encounters.

In the ASH technique the time differences between the two (or more) photons is calculated. If this time difference is more than $T_{R}$ then this is counted as a successful heralding event and the efficiency is calculated as in the previous case. Again in these heralding events the number of photons following the trigger photon is counted $\left(N_{2}\right.$ if there is 2 and $N_{1}$ if there is 1 ) and the purity can be calculated using the same formula as above as a function of the filtering time.

\section{II.D. Collection efficiency estimation}

To estimate the collection efficiency in our setup we take into account the efficiencies of the various optical components using three techniques:

- Simulation (sim) of collection efficiency into objective by using a commercial FDTD software (Lumerical)

- Transmission/Reflection measurement using a $655 \mathrm{~nm}$ diode laser (meas).

- Factory data (fact)

The efficiency of our system is estimated in table S2.

\section{II.E. Effect of noise on single photon purity}

Experimentally we expect that the sole source reducing $S$ in the signal port is from noise. To check this point we show the purity, $S$, of both schemes as a function of the filtering time $T_{F}$ after the excitation pulse. We can see from figure 3e in the main text that the ASH purity improves dramatically with even $1 \mathrm{~ns}$ of filtering which fits well with the extracted correlated noise lifetime. The TIMED scheme, on the other hand, is hardly affected by the filtering. This difference in sensitivity can be understood since in the TIMED scheme we apply a passive gate, so all short-lifetime counts will by default be directed to the idler port without affecting the fraction of photons arriving to the signal port. On the other hand, in the ASH scheme a short lifetime count will lead to premature "switching" to the signal port causing both bi-exciton and exciton photons to be directed to the signal port. To confirm that that the short-lifetime counts are indeed from correlated noise counts and not from higher order multiexcitons we estimate their rate based on the measured ASH purity at $T_{F}=0$. This is equivalent to finding the ratio of three photon to two photon events in the raw data. If indeed correlated noise is the source for the reduced $T_{F}=0$ purity, this ratio should give the probability per pulse to get a correlated noise count which turns out to be $1.4 \times 10^{-3}$ or correspondingly the rate is $\sim 2800 \mathrm{cps}$. This agrees with the correlated noise level measured at the same excitation power on the same substrate in a region with no quantum dots $(\sim 2500-3500 \mathrm{cps})$. For $T_{F}>1 \mathrm{~ns}, S$ reaches a maximum of around 0.995 , after filtering out nearly all correlated noise. Uncorrelated noise is what limits this value from reaching unity. Using the procedure as above we find that this noise rate is around $880 \mathrm{cps}$ which is in agreement with our uncorrelated noise rate $\sim 600-800$ cps. 
To confirm these statements we will attempt to reconstruct the measured purities based on the error rates stated above. In general the single photon purity of a signal can be defined as:

$$
S=\frac{P_{1}}{P_{\geq 1}}=1-\frac{P_{\geq 2}}{P_{\geq 1}}
$$

where $P_{1}, P_{\geq 1}$ and $P_{\geq 2}$ are the probabilities per relevant event of obtaining a single photon count, at least one photon , and at least two photons respectively.

Due to the low quantum yields in our case we can assume that $P_{\geq 2} \approx P_{2}$ and therefore:

$$
S=1-\frac{P_{2}}{P_{\geq 1}}
$$

Note the absence of the $\alpha$ factor here since we are theoretically considering the events obtained directly from the emitter. Now assume we have a certain probability per pulse $\eta_{c n}=1.4 \times 10^{-3} / \alpha$ and $\eta_{u n}=4.4 \times 10^{-4} / \alpha$ of obtaining correlated and uncorrelated noise event respectively. We will now study the effect of these noise terms on our measured purity:

\section{Heralded schemes}

For the heralded schemes in principle the purity should be unity unless there is noise. $P_{2}$ and $P_{\geq 1}$ are the probabilities of getting two photons and at least one photon conditioned on the presence of another trigger photon. For simplicity lets consider the ASH technique with $T_{R}=0$ in two regimes:

- In the presence of correlated noise $\left(T_{F}=0\right)$ in this case $P_{\geq 1} \approx Q Y_{X} Q Y_{B X}+\left(\eta_{c n}+\eta_{u n}\right)\left(Q Y_{X}+Q Y_{B X}\right)$ notice that due to the heralding requirement this is effectively the probability of two photons in a non heralding scheme. On the other hand the probability of obtaining two photons in the signal port of a heralding technique is just the correlation between the noise counts and the heralded counts given by $P_{2}=Q Y_{X} Q Y_{B X}\left(\eta_{c n}+\eta_{u n}\right)$. Therefore by using the values obtained from our experiment we find that the purity is given by:

$$
S_{\text {with noise }}=1-\frac{Q Y_{X} Q Y_{B X}\left(\eta_{c n}+\eta_{u n}\right)}{Q Y_{X} Q Y_{B X}+\left(\eta_{c n}+\eta_{u n}\right)\left(Q Y_{X}+Q Y_{B X}\right)}=0.9867
$$

- In the absence of correlated noise $\left(T_{F} \gg \tau_{c n}\right)$ and assuming that $T_{F}$ is still much shorter than the biexciton lifetime then we can effectively set $\eta_{c n}=0$ in the previous equation to obtain:

$$
S_{\text {withnoise }}=1-\frac{Q Y_{X} Q Y_{B X} \eta_{u n}}{Q Y_{X} Q Y_{B X}+\eta_{u n}\left(Q Y_{X}+Q Y_{B X}\right)}=0.996
$$

This is in good agreement with the results shown in figure $3 e$ of the main text.

\section{PRACTICAL DETAILS}

In this section we will discuss in detail the effect of response time on the performance of ASH. The diagram of the proposed optical and electronic circuit needed to implement this technique is displayed S3a and b. We conduct a detailed analysis of the different components that will contribute to $T_{R}$ in table S3 where the propagation delay of the $\mathrm{S} / \mathrm{R}$ latch is the composite delay of two NOR gates [6]. We consider different situations with either single photon avalanche detectors (SPAD) or semiconductor nanowire single photon detectors (SNSPD) combined with the other components either on-chip or free space. We compare the efficiency and single photon rate of the resulting 4 scenarios in Fig. S3c and d with a comparison with TIMED. Depending on the specific implementation the ASH technique will 
(a)
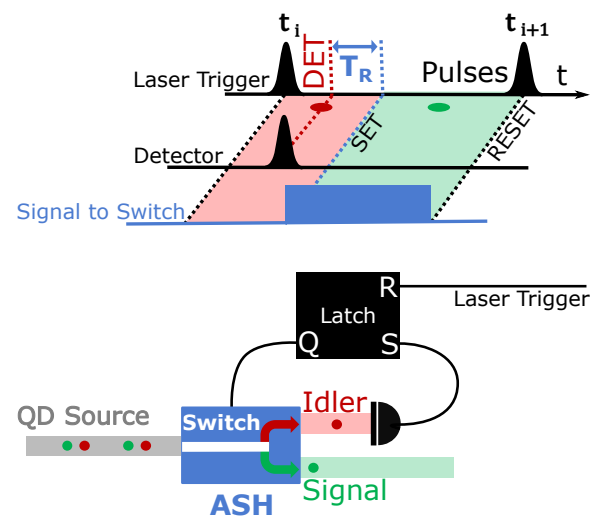

(b)

S/R Latch

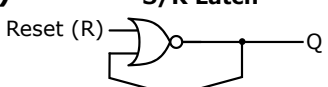

Set $(\mathrm{S}) \longrightarrow$
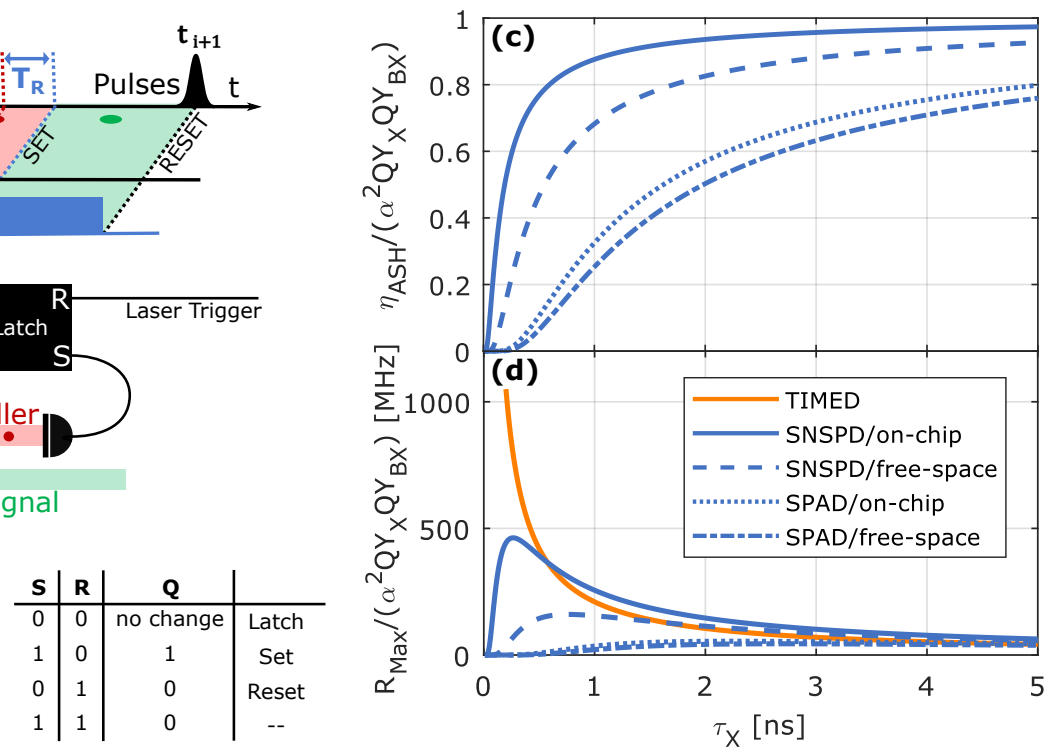

FIG. S3. Schemes for the (a) ASH scheme and (b) S/R latch. (c) The heralding efficiency and (d) single photon rate of ASH under different response times as compared to the TIMED technique

(a)

\begin{tabular}{|c|c|c|c|}
\hline Circuit Component & Type & Description & Value \\
\hline \hline \multirow{3}{*}{ Detector } & \multirow{2}{*}{ SNSPD } & Latency & $\sim 50 \mathrm{ps}[2]$ \\
\cline { 3 - 4 } & & Jitter & $15 \mathrm{ps}[3]$ \\
\cline { 2 - 4 } & \multirow{2}{*}{ SPAD } & Latency & $\sim 2 \mathrm{~ns} \mathrm{[4]}$ \\
\cline { 2 - 4 } & & Jitter & $50 \mathrm{ps} \mathrm{[5]}$ \\
\hline Logic Circuit & S/R Latch & Propagation delay & $185 \mathrm{ps}[6]$ \\
\hline Optical Switch & $25 \mathrm{GHz}$ Modulator & Rise time & $15 \mathrm{ps} \mathrm{[7]}$ \\
\hline \multirow{2}{*}{ Optical/Electronic Propagation } & On Chip & & Negligible \\
\cline { 2 - 4 } & Free Space & & $500 \mathrm{ps}$ \\
\hline
\end{tabular}

(b)

\begin{tabular}{c|c|c|} 
& Free Space & On-Chip \\
\hline \hline SNSPD & $765 \mathrm{ps}$ & $265 \mathrm{ps}$ \\
\hline SPAD & $2.75 \mathrm{~ns}$ & $2.25 \mathrm{~ns}$ \\
\hline
\end{tabular}

TABLE S3. (a) Contributions of various components to $T_{R}$. (b) The total response time under different configurations. .

be better than TIMED for $\tau_{X}$ more than a certain value.

[1] K. Matsuzaki, S. Vassant, H.-W. Liu, A. Dutschke, B. Hoffmann, X. Chen, S. Christiansen, M. R. Buck, J. A. Hollingsworth, S. Götzinger, and V. Sandoghdar, "Strong plasmonic enhancement of biexciton emission: controlled coupling of a single quantum dot to a gold nanocone antenna," Scientific Reports, vol. 7, p. 42307, 22017.

[2] J. P. Allmaras, A. G. Kozorezov, B. A. Korzh, K. K. Berggren, and M. D. Shaw, "Intrinsic timing jitter and latency in superconducting single photon nanowire detectors," 42018.

[3] Single Quantum, "Single Quantum Eos."

[4] I. Prochazka, J. Blazej, and J. Kodet, "Measurement of the optical to electrical detection delay in the detector for groundto-space laser time transfer," Metrologia, vol. 48, pp. L13-L16, 62011.

[5] PicoQuant GmbH, "PDM Series."

[6] ON Semiconductor, "2.5V/3.3V $12 \mathrm{~Gb} / \mathrm{s}$ Differential Clock/Data SmartGate."

[7] iXblue, "NIR-MX800-LN-10." 